\title{
Detection of a $\gamma$-ray halo around Geminga with the Fermi-LAT and implications for the positron flux
}

\author{
Mattia Di Mauro ${ }^{1,2}$, Silvia Manconi ${ }^{* 3,4}$, Fiorenza Donato ${ }^{3,4}$ \\ ${ }^{1}$ NASA Goddard Space Flight Center, Greenbelt, MD 20771, USA \\ ${ }^{2}$ Catholic University of America, Department of Physics, Washington DC 20064, USA \\ ${ }^{3}$ Dipartimento di Fisica, Università di Torino, via P. Giuria 1, 10125 Torino, Italy \\ ${ }^{4}$ Istituto Nazionale di Fisica Nucleare, Sezione di Torino, Via P. Giuria 1, 10125 Torino, Italy \\ E-mail: manconi@to.infn.it
}

\begin{abstract}
An excess of cosmic-ray positrons above $10 \mathrm{GeV}$ with respect to the spallation reaction of cosmic rays (CRs) with the interstellar medium has been measured by Pamela, Fermi-LAT and with unprecedented precision by AMS-02. Various interpretations have been invoked to interpret this excess, such as production from supernova remnants, pulsar wind nebulae (PWNe) and dark matter. A dominant contribution from dark matter is ruled out by the bounds found in gamma rays and other indirect searches. Models where supernova remnants produce secondary CRs struggle to explain the observed CR fluxes by AMS-02. Finally, severe constraints for a significant PWN contribution come from the detection of very high-energy gamma-ray emission from Monogem and Geminga PWNe by Milagro and HAWC experiments. In this contribution we present a detailed study of the GeV gamma-ray halo around Geminga and Monogem, and show the constraints found for the contribution of these PWNe to the cosmic-ray positron excess, combining Milagro and HAWC data with measurements from the Fermi-LAT for the first time. We report the detection of a significant emission from Geminga PWN, derived by including the proper motion of its pulsar. We demonstrate that using gamma-ray data from the LAT is of central importance to provide a precise estimate for a PWN contribution to the cosmic positron flux.
\end{abstract}

36th International Cosmic Ray Conference -ICRC2019-

July 24th - August 1st, 2019

Madison, WI, U.S.A.

${ }^{*}$ Speaker. 


\section{Introduction}

The flux of cosmic-ray electrons and positrons $\left(e^{ \pm}\right)$is now known with unprecedented precision from about $0.1 \mathrm{GeV}$ up to $\mathrm{TeV}$ energies, thanks to the data provided in the last few years by PAMELA, Fermi-LAT, AMS-02, DAMPE and CALET experiments $[1,2,3,4,5]$. The interpretation of these data is still debated, and is of central importance to reach a full understanding of the cosmic-ray acceleration and propagation in the Galaxy.

Among the different leptonic fluxes, the origin of the antimatter component (i.e., the $e^{+}$flux) has received particular attention. In fact, the $e^{+}$observed above $10 \mathrm{GeV}$ cannot be explained by the tipycal expectations from the secondary production, i.e. the $e^{+}$produced by spallation reactions of primary cosmic rays with the Interstellar Medium (ISM) [6]. This excess of cosmic-ray $e^{+}$has been intepretated invoking different mechanisms ( see [7, 8, 9] for recent reviews), such as the pairs emitted by pulsars and their Pulsar Wind Nebulae (PWNe) [10, 11, 12], the secondary emission in Supernova Remnants (SNRs)[13], modifications in the secondary production mechanism [14], or the annihilation or decay of dark matter particles in our Galaxy (see $[15,16,17]$ and references therein). However, a dominant contribution from dark matter is disfavoured, given the bounds obtained in other indirect searches [18]. The secondary emission in SNRs has been demonstrated to possibly produce at most only the $\sim 25 \%$ of the observed $e^{+}$fraction [19].

On the other hand, the idea that pulsars might be factories of cosmic-ray $e^{ \pm}$in our Galaxy dates back to 30-40 years ago [20, 21]. Multiwavelength observations of several PWNe confirm the presence of very-high energy $e^{ \pm}$pairs [22]. The spectral energy distribution (SED) of these objects, from radio to $\gamma$-ray energies, provides valuable information about the population of $e^{ \pm}$ produced by these sources [8]. Nevertheless, the details of the acceleration and release of pairs from PWNe in the interstellar medium are still under investigation, and are of major interest for assessing a possible contribution of PWNe to the cosmic-ray $e^{ \pm}$detected at Earth.

Recently, the Milagro and HAWC experiments have reported the detection of an extended $\gamma$ ray emission at energies larger than $5 \mathrm{TeV}$ from the direction of Geminga and Monogem PWNe, with an angular size of about $2^{\circ}[23,24]$. Geminga and Monogem (or J0633+1746 and B0656+14) pulsars are among the closest (distances of $0.250 \mathrm{kpc}$ and $0.288 \mathrm{kpc}$, and ages of $342 \mathrm{kyr}$ and $111 \mathrm{kyr}$, respectively) and most powerful sources in the ATNF catalog [25]. With a spin-down power of $W_{0} \sim 3 \times 10^{34} \mathrm{erg} \mathrm{s}^{-1}$, they have been considered for long among the main candidates to contribute to the $e^{+}$flux at Earth $[26,27,28]$. These $\gamma$-ray measurements can be used to precisely quantify the contribution of these PWNe to the cosmic-ray $e^{+}$flux at Earth $[29,30,31,32]$. In fact, the extended $\mathrm{TeV} \gamma$-ray emission seen by HAWC and MILAGRO has been interpreted as inverse Compton scattering (ICS) emission of $e^{ \pm}$accelerated, and then released, by these sources and interacting with the interstellar radiation field (ISRF) [33]. The ISRF, composed of the cosmic microwave background (CMB), infrared (IR) and starlight (SL), is then scattered up to $\gamma$-ray energies. The angular extension of this $\mathrm{TeV} \gamma$-ray emission, together with the age of the sources, suggest that these ICS photons are produced by $e^{ \pm}$pairs escaped from the PWNe, at a distance of few tens of parsec. However, the $\gamma$ rays between $5-40 \mathrm{TeV}$ detected by HAWC are produced via ICS off the ISRF by $e^{ \pm}$at average energies of at least tens of TeV. Since the $e^{+}$AMS-02 excess is between a few tens up to hundreds of $\mathrm{GeV}$, the HAWC data cannot test directly the origin of this excess. The use of HAWC $\gamma$-ray data in order to predict the $e^{+}$flux at AMS-02 energies is indeed an extrapo- 
lation, which can affect significantly the conclusion on the $e^{+}$flux, depending on the assumptions made. Moreover, data from the Fermi-LAT experiment in the energy range of $10-1000 \mathrm{GeV}$ are perfectly suited in order to constrain more precisely the Monogem and Geminga contribution to the $e^{+}$at $E>100 \mathrm{GeV}$, since ICS photons in this energy range are produced by $e^{ \pm}$detected at Earth with average energies in the range $350-1500 \mathrm{GeV}$.

In this contribution we discuss the search for an extended $\gamma$-ray emission in the direction of Geminga and Monogem using Fermi-LAT data, which can be attributed to the ICS of the accelerated $e^{ \pm}$pairs off the ISRF.

\section{Positron and photon flux from PWNe}

We here summarize the basic elements of our model for the emission of $e^{ \pm}$and $\gamma$-rays from PWNe, which is carefully illustrated in Ref. [34].

The $e^{ \pm}$that propagate in the Galaxy produce $\gamma$ rays through ICS with the Galactic ISRF, which is composed of the CMB, the IR light, and the SL. The ISRF energy density in the local Galaxy is taken as in [33]. We model the photon flux emitted for the ICS by the pulsar, at an energy $E_{\gamma}$ and coming from a solid angle $\Delta \Omega$, as [35, 36]:

$$
\phi^{\mathrm{IC}}\left(E_{\gamma}, \Delta \Omega\right)=\int_{m_{e} c^{2}}^{\infty} d E \mathscr{M}(E, \Delta \Omega) \mathscr{P}^{\mathrm{IC}}\left(E, E_{\gamma}\right) .
$$

The quantity $\mathscr{M}(E, \Delta \Omega)$ is the spectrum of $e^{+}$and $e^{-}$of energy $E$ which propagate in the Galaxy and from a solid angle $\Delta \Omega$, and $\mathscr{P}^{\mathrm{IC}}\left(E, E_{\gamma}\right)$ is the power of photons emitted by a single $e^{ \pm}$for ICS, defined as in [35, 37].

Highly energetic $e^{ \pm}$pairs are believed to be produced in PWNe under the influence of winds and shocks around the pulsars, then accelerated up to very high energies, and finally injected into the ISM, typically after a few tens of kyr $[20,11]$. We here consider a continuous injection scenario to describe the emission mechanism of $e^{ \pm}$in PWNe, where the particles are emitted with a rate that follows the pulsar spin-down energy, which is translated in the energy of $e^{ \pm}$pairs with an efficiency $\eta$. This time-dependent $e^{ \pm}$injection spectrum $Q(E, t)$ is obtained as described in Refs [38, 34]. The HAWC data suggest that the diffusion coefficient $\left(D(E)=D_{0}(E / 1 \mathrm{GeV})^{-\delta}\right)$ in the vicinity of Geminga and Monogem PWNe may be $\sim 500$ times smaller than the one usually derived for the average of the Galaxy [24]. We take into account this observation by using a two-zone diffusion model $[32,39,40]$, where the region of inefficient diffusion is contained around the source, and delimited by an empirical radius $r_{b}$. For this two-zone diffusion model, we use the definition of the diffusion coefficient, as well as the solution for the $e^{ \pm}$density at the Earth position, as derived in Ref. [39]. As for the regions in the Galaxy for $r>r_{b}$, we use the propagation parameters obtained in [41] $\left(\mathrm{K} 15, D_{0}=0.0967 \mathrm{kpc}^{2} / \mathrm{Myr}, \delta=0.408\right)$ and [42] $\left(\mathrm{G} 15, D_{0}=0.05 \mathrm{kpc}^{2} / \mathrm{Myr}, \delta=0.445\right)$.

\section{Analysis setup}

The point-like pulsed emissions from the Monogem and Geminga pulsars is included in FermiLAT source catalogs [43] ${ }^{1}$. A multiple-degree extended emission has instead never been claimed.

\footnotetext{
${ }^{1}$ See https://fermi.gsfc.nasa.gov/ssc/data/access/lat/8yr_catalog/for the most recent Fermi-LAT source catalog obtained with 8 years of data.
} 


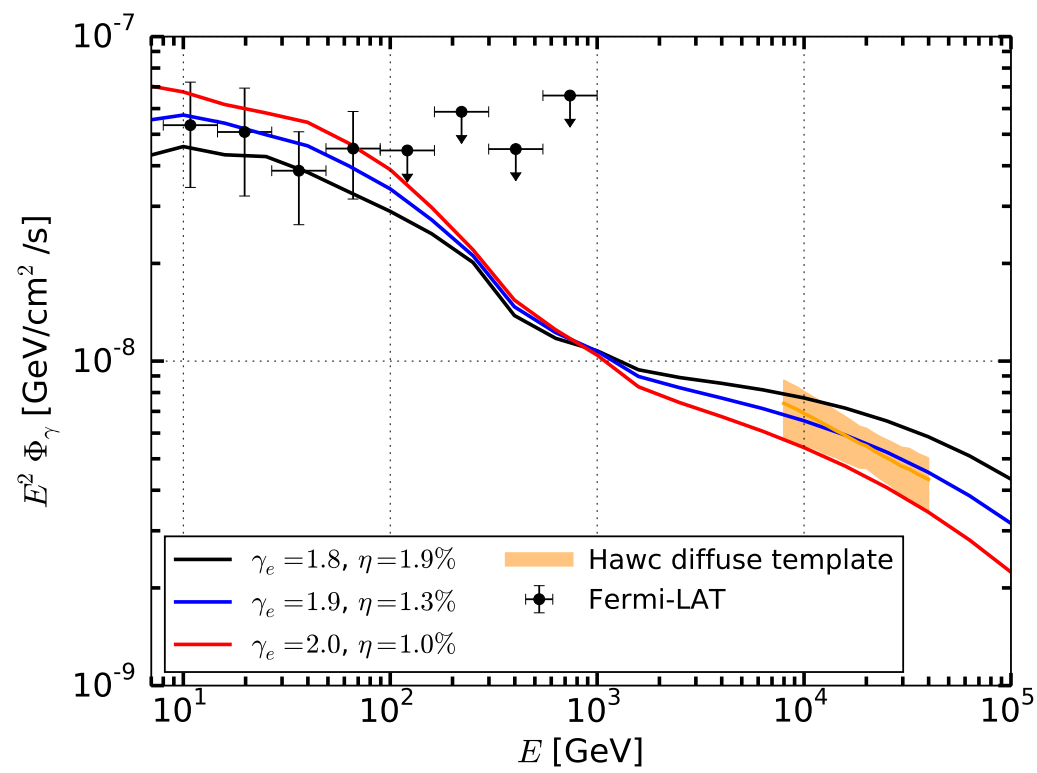

Figure 1: The $\gamma$-ray flux for ICS from Geminga. The Fermi-LAT data we derived are shown as black dots. We report the HAWC data (obtained using a diffuse template) as an orange band [24]. The curves are the flux predictions obtained for different values of $\gamma_{e}$ and $\eta$.

In order to search for such a signal, we analyze 115 months of Fermi-LAT Pass 8 data, in the energy range $E=[8,1000] \mathrm{GeV}$, passing standard data quality selection criteria, belonging to the Pass 8 SOURCE event class, and using the instrument response functions P 8R3_SOURCE_V2. We consider energies above $8 \mathrm{GeV}$, because at lower energies the interstellar emission model (IEM), as well as the pulsed emission from the pulsar, dominate the $\gamma$-ray data. Our region of interest (ROI) is of $70^{\circ} \times 70^{\circ}$, and it is centered at RAJ2000 $=95^{\circ}$ and DEJ2000 $=13^{\circ}$. The data are binned with a pixel size of $0.06^{\circ}$, and 6 bins per energy decade.

We expect that the morphology of ICS emission is energy dependent. In particular, the larger the value of $D_{0}$, the more extended will be the ICS emission. For example, for $D_{0}=1.5 \cdot 10^{26} \mathrm{~cm}^{2} / \mathrm{s}$, and for $E_{\gamma}<40 \mathrm{GeV}$, we estimate the angle containing the $68 \%$ of the total ICS $\gamma$-ray emission (i.e the extension) to be $\sim 10^{\circ}$. The extension decreases significantly for higher energies, and is about $3^{\circ}$ in the HAWC energy range. The energy dependence of the spatial morphology of the ICS emission is taken into account by creating a mapcube template, a three dimensional table that, for each energy bin, gives the $\gamma$-ray intensity in Galactic longitude and latitude. For simplicity, we assume a one-zone diffusion model for the $\gamma$-ray ICS halo. This is a reasonable choice, since for the energies considered in our analysis the low-diffusion zone dominates our ROI. In addition, we include the effect on the ICS $\gamma$-ray morphology coming from the proper motion of the Geminga pulsar, which is relevant for energies below a few hundred $\mathrm{GeV}[39,34]$. In fact, the Geminga pulsar has a proper motion of $178.2 \pm 1.8$ mas/year, corresponding to a transverse velocity of $v_{T} \approx 211(d / 250 \mathrm{pc}) \mathrm{km} \mathrm{s}^{-1}$ [44].

Our model fit to the data includes the IEM (with free normalization and spectral shape), the isotropic template (with free normalization) and cataloged sources (with free normalization and 
spectral shape) from the preliminary 8 years list ${ }^{2}$. We employed the IEM released with Pass 8 data [45] (i.e., gll_iem_v06.fits). We also repeated the analysis using 10 different IEM (see [34]), in order to derive the systematics in the result associated to this choice. As for the templates of the Monogem and Geminga ICS halos, we vary $D_{0}$ in the range $10^{25}-10^{29} \mathrm{~cm}^{2} / \mathrm{s}$, as well as their spectral slope, and perform our analysis for different values of $D_{0}$.

\section{Results}

We detect the Geminga ICS halo in Fermi-LAT data with $T S=65-143^{3}$ and $D_{0}=1.6-3.5$. $10^{26} \mathrm{~cm}^{2} / \mathrm{s}$, depending on the considered IEM. The value we find for $D_{0}$ is compatible within $2 \sigma$ errors with the result by the HAWC Collaboration $\left(D_{0}=6.9_{-2.2}^{+3.0} \cdot 10^{25} \mathrm{~cm}^{2} / \mathrm{s}{ }^{4}\right)$. In addition, our analysis significantly detects the motion of Geminga pulsar by fitting its ICS halo. In fact, the fit in which we include the effect of the proper motion in the ICS template is preferred at 4.7-7.1 $\sigma$, depending on the IEM model. The Monogem halo is not detected in Fermi-LAT data, regardless of the value of $D_{0}$. We derive the $95 \%$ lower limit on the value of the diffusion coefficient to be $D_{0}>1-10 \cdot 10^{26} \mathrm{~cm}^{2} / \mathrm{s}$, which is compatible with Ref. [24].

The flux values for the Geminga ICS halo are reported in Fig. 1. They are evaluated independently in different energy bins, by leaving free to vary the SED parameters of the sources in the model, as well as of the IEM and the isotropic templates. The Fermi-LAT measures the Geminga ICS halo with a precision of about $30 \%$ from $8 \mathrm{GeV}$ up to $100 \mathrm{GeV}$. As for the remaining explored energies, we obtain upper limits. We also report our predictions for the SED derived using the modeling described in Sec. 2. By fitting the Fermi-LAT data, we derive the efficiency of spin-down energy conversion $(\eta)$ for different $e^{+}$spectral indices. For $\gamma_{e}=[1.8,1.9,2.0]$, we find $\eta=[0.019,0.013,0.010]$, respectively. We note that the chosen $\gamma_{e}$ values bracket the HAWC measurements. An analogous analysis for Monogem for $\gamma_{e}=1.9$ and 2.1 results in $\eta \leq 0.008$ and 0.006 , respectively.

We now use our findings to predict the contribution of Geminga and Monogem to the $e^{+}$ flux at Earth. The $e^{+}$flux is computed implementing the $\eta$ fitted on the Fermi-LAT data, for the different $e^{+}$spectral indices. Since the $e^{+}$emitted from the Geminga and Monogem PWNe travel in both the low and high-diffusion zones before reaching the Earth, a two-zone diffusion model is used (see Sec. 2 and reference therein). The results are shown in Fig. 2 for $r_{b}=100$ pc, and using for $r>r_{b}$ the K15 and G15 Galactic propagation models. The different $\gamma_{e}$ and $\eta$ give very similar predictions at hundreds of $\mathrm{GeV}$ up to $\mathrm{TeV}$ energies, where the Fermi-LAT $\gamma$ rays calibrate the progenitor leptons. Therefore, at lower $e^{+}$energies softer injection spectra give higher $e^{+}$flux. The Geminga PWN, as constrained now by Fermi-LAT data, contributes at a few per-cent level to the positron flux at $100 \mathrm{GeV}$. The highest contribution from Geminga is about $10 \%$ of the last AMS-02 energy data point at around $800 \mathrm{GeV}$. As for Monogem (not present in this figure, but see [34]), it can produce at most $3 \%$ of the flux at the highest energy measured $e^{+}$flux. Additional tests

\footnotetext{
${ }^{2}$ https://fermi.gsfc.nasa.gov/ssc/data/access/lat/fl8y/gll_psc_8year_v5.fit

${ }^{3}$ (Test Statistic (TS) defined as twice the difference in maximum log-likelihood between the null hypothesis (i.e., no source present) and the test hypothesis: $T S=2\left(\log \mathscr{L}_{\text {test }}-\log \mathscr{L}_{\text {null }}\right)$.)

${ }^{4}$ obtained by rescaling HAWC diffusion coefficient for electrons at $100 \mathrm{TeV}, D_{100}$, to $D_{0}$ for $\delta=1 / 3$
} 


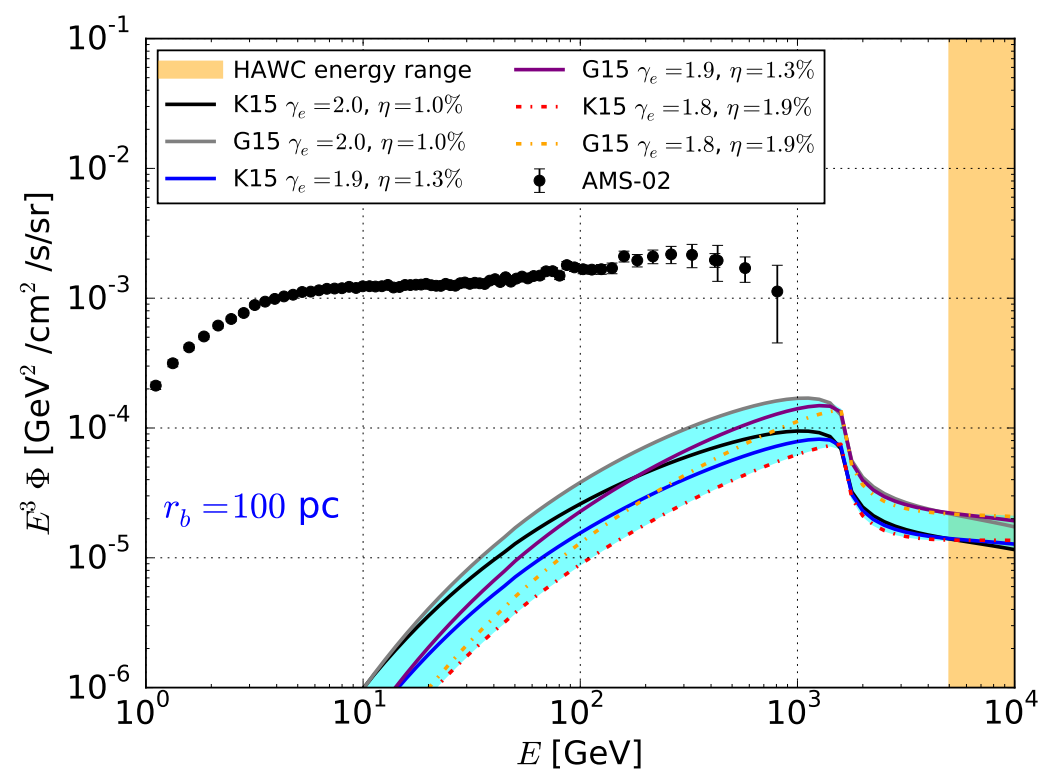

Figure 2: $e^{+}$flux at Earth from Geminga as computed within a two-zone diffusion model, and for the $\gamma_{e}$, $\eta$ values compatible with Fermi-LAT data. Blue (purple) curves are for G15 (K15) propagation model and for $r_{b}=100 \mathrm{pc}$. The cyan band embeds the differences in the results considering these two propagation parameters and the choice of $\gamma_{e}$.

that validate the detection of the Geminga ICS halo in Fermi-LAT data against different systematics are discussed in Ref. [34].

\section{Conclusions}

We reported the first detection of a counterpart of the Geminga $\gamma$-ray halo seen by HAWC in Fermi-LAT data from $8 \mathrm{GeV}$ up to hundreds of $\mathrm{GeV}$ [34]. As for Monogem, we derived stringent upper limits. We accurately modeled the ICS emission from $e^{ \pm}$pairs produced in PWNe, as well as the effects of the proper motion of Geminga pulsar, as this affects the spatial morphology of the ICS $\gamma$-ray halo at GeV energies. We demonstrated that using Fermi-LAT data, together with HAWC measurements, can significantly constrain the $e^{+}$flux from these two sources. We conclude that these sources alone, as bound now by Fermi-LAT data, cannot be the major contributors to the $e^{+}$excess. However, a Galactic population of pulsars with efficiency in the range of $1-3 \%$ and physical spin-down properties has been recently demonstrated to explain the $e^{+}$flux excess [46]. This result, together with the results discussed in [12] for cataloged pulsars, suggest that the cumulative $e^{+}$emission from Galactic PWNe remains a viable interpretation for the $e^{+}$excess.

\section{Acknowledgments}

The Fermi LAT Collaboration acknowledges generous ongoing support from a number of agencies and institutes that have supported both the development and the operation of the LAT as well as scientific data analysis. These include the National Aeronautics and Space Administration 
and the Department of Energy in the United States, the Commissariatá l'Energie Atomique and the Centre National de la Recherche Scientifique / Institut National de Physique Nucléaire et de Physique des Particules in France, the Agenzia Spaziale Italiana and the Istituto Nazionale di Fisica Nucleare in Italy, the Ministry of Education, Culture, Sports, Science and Technology (MEXT), High Energy Accelerator Research Organization (KEK) and Japan Aerospace Exploration Agency (JAXA) in Japan, and the K. A. Wallenberg Foundation, the Swedish Research Council and the Swedish National Space Board in Sweden. Additional support for science analysis during the operations phase is gratefully acknowledged from the Istituto Nazionale di Astrofisica in Italy and the Centre National d'Etudes Spatiales in France. This work performed in part under DOE Contract DE- AC02-76SF00515.

MDM acknowledges support by the NASA Fermi Guest Investigator Program 2014 through the Fermi multi-year Large Program N. 81303 (P.I. E. Charles). The work of FD and SM is supported by the "Departments of Excellence 2018 - 2022" Grant awarded by the Italian Ministry of Education, University and Research (MIUR) (L. 232/2016). FD and SM acknowledge financial contribution from the agreement ASI-INAF n.2017-14-H.0 and the Fondazione CRT for the grant $2017 / 58675$.

\section{References}

[1] O. Adriani et al., Nature 458, pp. 607-609 (2009).

[2] M. Ackermann, M. Ajello, Allafort, et al., Physical Review Letters 108(1), pp. 011103 (2012).

[3] M. Aguilar, L. Ali Cavasonza, G. Ambrosi, et al., Phys. Rev. Lett. 122, pp. 041102 (2019).

[4] G. Ambrosi et al., Nature 552, pp. 63-66 (2017).

[5] O. Adriani, Y. Akaike, K. Asano, et al., Phys. Rev. Lett. 120, pp. 261102 (2018).

[6] T. Delahaye, F. Donato, N. Fornengo, et al., Astron. Astrophys. 501, pp. 821-833 (2009).

[7] Pasquale D. Serpico, Astropart. Phys. 39-40, pp. 2-11 (2012).

[8] A. M. Bykov, E. Amato, A. E. Petrov, et al., Space Sci. Rev. 207(1-4), pp. 235-290 (2017).

[9] Pasquale Dario Serpico, J. Astrophys. Astron. 39, pp. 41 (2018).

[10] Dmitry Malyshev, Ilias Cholis, and Joseph Gelfand, Phys. Rev. D80, pp. 063005 (2009).

[11] P. Blasi and E. Amato, Astrophysics and Space Science Proceedings 21, pp. 624 (2011).

[12] M. Di Mauro, F. Donato, N. Fornengo, et al., JCAP 4, pp. 6 (2014).

[13] P. Blasi, Physical Review Letters 103(5), pp. 051104 (2009).

[14] Nicola Tomassetti and Fiorenza Donato, Astrophys. J. 803(2), pp. L15 (2015).

[15] Lars Bergstrom, Torsten Bringmann, and Joakim Edsjo, Phys. Rev. D78, pp. 103520 (2008).

[16] Gianfranco Bertone, Marco Cirelli, Alessandro Strumia, et al., JCAP 0903, pp. 009 (2009).

[17] M. Di Mauro, F. Donato, N. Fornengo, et al., JCAP 5, pp. 031 (2016).

[18] Marco Cirelli and Paolo Panci, Nucl. Phys. B821, pp. 399-416 (2009).

[19] I. Cholis and D. Hooper, Phy. Rev. D 89(4), pp. 043013 (2014). 
[20] X. Chi, K. S. Cheng, and E. C. M. Young, ApJL 459, pp. L83 (1996).

[21] A. Boulares, ApJ 342, pp. 807-813 (1989).

[22] Bryan M. Gaensler and Patrick O. Slane, Ann. Rev. Astron. Astrophys. 44, pp. 17-47 (2006).

[23] A. A. Abdo, B. T. Allen, T. Aune, et al., ApJL 700, pp. L127-L131 (2009).

[24] A. U. Abeysekara et al., Astrophys. J. 843(1), pp. 40 (2017).

[25] R. N. Manchester, G. B. Hobbs, A. Teoh, et al., AJ 129, pp. 1993-2006 (2005).

[26] Dan Hooper, Pasquale Blasi, and Pasquale Dario Serpico, JCAP 0901, pp. 025 (2009).

[27] Tim Linden and Stefano Profumo, Astrophys. J. 772, pp. 18 (2013).

[28] Silvia Manconi, Mattia Di Mauro, and Fiorenza Donato, JCAP 1701(01), pp. 006 (2017).

[29] H. Yüksel, M. D. Kistler, and T. Stanev, Physical Review Letters 103(5), pp. 051101 (2009).

[30] A. U. Abeysekara et al., Science 358(6365), pp. 911-914 (2017).

[31] Dan Hooper, Ilias Cholis, Tim Linden, et al., Phys. Rev. D96(10), pp. 103013 (2017).

[32] Stefano Profumo, Javier Reynoso-Cordova, Nicholas Kaaz, et al., Phys. Rev. D97(12), pp. 123008 (2018).

[33] Silvia Vernetto and Paolo Lipari, Phys. Rev. D94(6), pp. 063009 (2016).

[34] Mattia Di Mauro, Silvia Manconi, and Fiorenza Donato, (arXiv:1903.05647).

[35] G. R. Blumenthal and R. J. Gould, Reviews of Modern Physics 42, pp. 237-271 (1970).

[36] Marco Cirelli, Gennaro Corcella, Andi Hektor, et al., JCAP 1103, pp. 051 (2011), [Erratum: JCAP1210,E01(2012)].

[37] T. Delahaye, J. Lavalle, R. Lineros, et al., A\&A 524, pp. A51 (2010).

[38] Hasan Yuksel, Matthew D. Kistler, and Todor Stanev, Phys. Rev. Lett. 103, pp. 051101 (2009).

[39] Xiaping Tang and Tsvi Piran, Mon. Not. Roy. Astron. Soc. 484(3), pp. 3491-3501 (2019).

[40] C. Evoli, T. Linden, and G. Morlino, ArXiv e-prints (2018).

[41] Rolf Kappl, Annika Reinert, and Martin Wolfgang Winkler, JCAP 1510(10), pp. 034 (2015).

[42] Y. Genolini, A. Putze, P. Salati, et al., Astron. Astrophys. 580, pp. A9 (2015).

[43] M. Ackermann et al., Astrophys. J. Suppl. 209, pp. 34 (2013).

[44] J. Faherty, F.M. Walter, and Anderson, Astrophysics and Space Science 308, pp. 225âĂŞ230 (2007).

[45] F. Acero et al., Astrophys. J. Suppl. 223(2), pp. 26 (2016).

[46] Ilias Cholis, Tanvi Karwal, and Marc Kamionkowski, Phys. Rev. D98(6), pp. 063008 (2018). 